\title{
TOEPLITZ ALGEBRAS ASSOCIATED WITH ENDOMORPHISMS AND PIMSNER-VOICULESCU EXACT SEQUENCES
}

\author{
M. KhoshKam And G. Skandalis
}

Let $A$ be a $C^{*}$-algebra and $\alpha$ a $*$-endomorphism of $A$. The analogue of Pimsner-Voiculescu exact sequences are obtained for the pair $(A, \alpha)$. We prove that the corresponding Toeplitz algebra remains KK-equivalent to $\mathrm{A}$.We also consider the situation where a semigroup $\left(\alpha^{t}\right)_{t \in \mathbb{R}_{+}}$of $*$-endomorphisms is acting on $A$ and formulate similar exact sequences. In this part we use the language of Connes-Higson E-theory.

\section{Introduction.}

One of the most celebrated results in the $K$-theory of $C^{*}$-algebras is the exact sequence proved by M. Pimsner and D. Voiculescu ([10]). This sequence allows one to compute the $K$-groups of a crossed product $C^{*}$-algebra $A \rtimes_{\alpha} \mathbb{Z}$, from a six term exact sequence involving $K$-groups of $A$, obtained from the $K$-theory sequence associated with an extension of $A \rtimes_{\alpha} \mathbb{Z}$ by $A \otimes K(H)$ where $\alpha \in \operatorname{Aut}(A)$. This extension referred to as the generalized Toeplitz extension, is given by a $C^{*}$-algebra denoted by $\mathcal{T}_{\alpha}$, called the Toeplitz algebra associated with the pair $(A, \alpha)$. Pimsner and Voiculescu proved that the natural inclusion of $A$ in $\mathcal{T}_{\alpha}$ induces an isomorphism at the level of $K$ groups. This allows one to obtain a six term exact sequence involving only the K-groups of $A$ and the crossed product $A \rtimes_{\alpha} \mathbb{Z}$. Later, in [5], using a generalization of Connes' "Thom isomorphism", T. Fack and G. Skandalis obtained the same exact sequence for $K K$-groups.

In this article we are concerned with extending Pimsner Voiculescu Exact Sequence to the situation where $\alpha$ is an Endomorphism. Our first task will be to define an appropriate notion of the Toeplitz algebra and an extension from which the $K$-theory sequence can be obtained. It is proved that this generalized Toeplitz algebra is still $K K$-equivalent to $A$. We then obtain similar results in the case of semigroups (indexed by $\mathbb{R}_{+}$) of endomorphisms.

While this work was almost finished, we received a remarkable preprint by Mihai Pimsner $([9])$, who considers the same Toeplitz algebra and proves the same extension and $K K$-theory results as ours in a much more general 
situation than that of a single endomorphism: Pimsner considers a Hilbert $A$-module $E$ which is 'generating' in that sense that the closed ideal spanned by the scalar products $\langle x, y\rangle, x, y \in E$ is $A$ itself and a morphism $\varphi$ from $A$ into $\mathcal{L}(E)$. An endomorphism is then just the particular case $E=A$ and $\varphi(A) \subset A=\mathcal{K}(A) \subset \mathcal{L}(A)=\mathcal{M}(A)^{1}$.

We think however that our paper may help understanding Pimsner's more general and interesting point of view. Moreover, our results may be used to give an alternate proof of Pimsner's (when $\varphi(A) \subset \mathcal{K}(E)$ ). Indeed, the condition on $E$ means that $\mathcal{K}(E)$ and $A$ are Morita equivalent; hence $\mathcal{K}(H) \otimes$ $\mathcal{K}(E)$ is isomorphic to $\mathcal{K}(H) \otimes A$ (at least in the separable case). We then get a morphism from $\mathcal{K}(H) \otimes A$ into itself which brings us to our case.

The organization of this paper is as follows.

- In Section 1, the Toeplitz algebra $\mathcal{T}_{\alpha}$ for a pair $(A, \alpha)$ with $\alpha \in \operatorname{End}(A)$ is defined and the basic properties are established. In particular, we show that $\mathcal{T}_{\alpha}$ is a full corner of a crossed product. This will be useful in realization of certain semigroup $C^{*}$-algebras.

- In Section 2, we deal with $K K$-groups and construction of an invertible element in the group $K K\left(A, \mathcal{T}_{\alpha}\right)$.

- Section 3 is concerned with extending our results of Sections (1) and (2) to a semigroup $\left(\alpha_{t}\right)_{t \in \mathbb{R}^{+}}$of endomorphisms of a $C^{*}$-algebra $A$. An appropriate notion of Toeplitz algebra is defined and the corresponding extension is formulated. In the continuous case, the Toeplitz algebra is $K$-Theoretically trivial.

One possible application for these results is in the study of semigroup $C^{*}$ algebras ([3]). From the basic theory if $S$ is a simple inverse semigroup, then it has a decomposition into a type of semi-direct product (known as Bruck Reilly product) of a group $G$ with the bicyclic semigroup $\mathcal{C}$. The action of $\mathcal{C}$ on $G$ is given by an endomorphism $\alpha$ of $G$. It can be proved that $C^{*}(S)$ the $C^{*}$-algebra of $S$ is $*$-isomorphic to the Toeplitz algebra associated with the pair $\left(C^{*}(G), \alpha\right)$. These ideas will be pursued elsewhere.

Finally, we point out that in ([4]), Ruy Excel obtains a generalization of Pimsner-Voiculescu Exact Sequence. But he considers a different situation dealing with ideals and $C^{*}$-algebras equipped with an action of $S$. The only overlap is that we both obtain Pimsner Voiculescu Exact Sequence as a special case. However, our methods are independent.

\footnotetext{
${ }^{1}$ Even in that case, our Toeplitz algebra differs slightly from Pimsner's. This will be explained at the end of the first section.
} 


\section{The Toeplitz algebra $\mathcal{T}_{\alpha}$.}

Notation. Recall that if $A$ is a $C^{*}$-algebra, $E, F$ are Hilbert $A$-modules, $x \in E$, and $y \in F$, we denote by $\theta_{x, y}: F \rightarrow E$ the operator $z \mapsto x\langle y, z\rangle$. An operator from $F$ to $E$ is said to be compact if it belongs to the closure $\mathcal{K}(F, E)$ of the vector space spanned by $\theta_{x, y}$ for $x \in E, y \in F$.

Let $A$ be a $C^{*}$-algebra and $\alpha$ an endomorphsm of $A$. Let $\mathcal{H}_{A}$ be the Hilbert $A$-module $\ell^{2}(\mathbb{N}, A)$, i.e., the set of sequences $\left(x_{n}\right)_{n \in \mathbb{N}}$ such that the series $\sum_{n \in \mathbb{N}} x_{n}^{*} x_{n}$ is norm convergent.

Let $S \in \mathcal{L}\left(\mathcal{H}_{A}\right)$ be the forward shift: i.e., $S\left(\left(x_{n}\right)_{n \in \mathbb{N}}\right)=\left(y_{n}\right)_{n \in \mathbb{N}} \in \mathcal{H}_{A}$ where, for $n \neq 0, y_{n}=x_{n-1}$ and $y_{0}=0$.

Define the faithful $*$-representation $\pi_{\alpha}$ of $A$ in $\mathcal{H}_{A}$ setting for $a \in A$ and $\left(x_{n}\right)_{n \in \mathbb{N}} \in \mathcal{H}_{A}$

$$
\pi_{\alpha}(a)\left(\left(x_{n}\right)_{n \in \mathbb{N}}\right)=\left(\alpha^{n}(a) x_{n}\right)_{n \in \mathbb{N}} \in \mathcal{H}_{A}
$$

For all $a \in A, \pi_{\alpha}(a) S=S \pi_{\alpha}(\alpha(a))$. It follows that the closed vector span of $\left\{S^{n} \pi_{\alpha}(a) S^{* m}: m, n \in \mathbb{N}, a \in A\right\}$ is a $C^{*}$-subalgebra of $\mathcal{L}\left(\mathcal{H}_{A}\right)$.

Definition 1.1. The $C^{*}$-subalgebra of $\mathcal{L}\left(\mathcal{H}_{A}\right)$ generated by $\left\{S^{n} \pi_{\alpha}(a) S^{* m}\right.$ : $m, n \in \mathbb{N}, a \in A\}$ is denoted by $\mathcal{T}_{\alpha}$ and is called the Toeplitz algebra associated with $(A, \alpha)$. We denote by $d_{\alpha}$ or just $d$ the morpism $\pi_{\alpha}$ as a morphism from $A$ to $\mathcal{T}_{\alpha}$.

If $A$ is unital and $\alpha(1)=1$, then $\pi_{\alpha}(1)$ is the identity element of $\mathcal{L}\left(\mathcal{H}_{A}\right)$, thus $S \in \mathcal{T}_{\alpha}$ and $\mathcal{T}_{\alpha}$ is the $C^{*}$-subalgebra of $\mathcal{L}\left(\mathcal{H}_{A}\right)$ generated by $S$ and $d_{\alpha}(A)$. In general, let $\tilde{A}$ be the $C^{*}$-algebra obtained from $A$ by adjoining an identity. Let $\tilde{\alpha}: \tilde{A} \rightarrow \tilde{A}$ be the unital extension of $\alpha$ to $\tilde{A}$. Then $\mathcal{T}_{\alpha}$ sits in $\mathcal{T}_{\tilde{\alpha}}$ as a two sided ideal.

The construction of the Toeplitz algebra $\mathcal{T}_{\alpha}$ satisfies the following naturality. Let $A$, and $B$ be $C^{*}$-algebras with endomorphisms $\alpha$ and $\beta$ respectively. To any $*$-homomorphism $\varphi: A \rightarrow B$ such that $\varphi \circ \alpha=\beta \circ \varphi$ there corresponds a $*$-homomorphism $\tau_{\varphi}: \mathcal{T}_{\alpha} \rightarrow \mathcal{T}_{\beta}$ given by $\tau_{\varphi}\left(S^{n} d_{\alpha}(a) S^{* m}\right)=$ $S^{n} d_{\beta}(\varphi(a)) S^{* m}$.

- If $A$ and $B$ are unital and $\varphi(1)=1$, we have an identification $\mathcal{H}_{A} \otimes_{A} B \cong$ $\mathcal{H}_{B}$ thus a morphism $\mathcal{L}\left(\mathcal{H}_{A}\right) \rightarrow \mathcal{L}\left(\mathcal{H}_{B}\right)$ which maps $\mathcal{T}_{\alpha}$ into $\mathcal{T}_{\beta}$.

- In particular, let $\varepsilon: \tilde{A} \rightarrow \mathbb{C}$ be the morphism with kernel $A$. Then $\tau_{\varepsilon}$ is a morphism from $\mathcal{T}_{\tilde{\alpha}}$ to the Toeplitz algebra $\mathcal{T}$ associated with the identity morphism of $\mathbb{C}$ whose kernel is $\mathcal{T}_{\alpha}$.

- To prove the existance of the morphism $\tau_{\varphi}$ in the general case, extend $\varphi$ to a unital morphism $\tilde{\varphi}: \tilde{A} \rightarrow \tilde{B}$; the corresponding morphism $\tau_{\tilde{\varphi}}: \mathcal{T}_{\tilde{\alpha}} \rightarrow \mathcal{T}_{\tilde{\beta}}$ maps $\mathcal{T}_{\alpha} \subset \mathcal{T}_{\tilde{\alpha}}$ into $\mathcal{T}_{\beta} \subset \mathcal{T}_{\tilde{\beta}}$.

Let us explore the structure of the Toeplitz algebra $\mathcal{T}_{\alpha}$ : 
Let $a, b \in A$ and $n, m \in \mathbb{N}$; let $\xi, \eta \in \mathcal{H}_{A}$ be the elements defined by $\xi(n)=a, \xi(k)=0$ for $k \neq n, \eta(m)=b$ and $\eta(k)=0$ for $k \neq m$. Then, $\theta_{\xi, \eta}=S^{n} d_{\alpha}\left(a b^{*}\right)\left(1-S S^{*}\right) S^{*^{m}} \in \mathcal{T}_{\alpha}$. Since the elements of the above form span $\mathcal{K}\left(\mathcal{H}_{A}\right)$, it follows that $\mathcal{K}\left(\mathcal{H}_{A}\right) \subset \mathcal{T}_{\alpha}$. As $\mathcal{T}_{\alpha} \subset \mathcal{L}\left(\mathcal{H}_{A}\right)=\mathcal{M}\left(\mathcal{K}\left(\mathcal{H}_{A}\right)\right)$ it follows that $\mathcal{K}\left(\mathcal{H}_{A}\right)=\mathcal{K}(H) \otimes A$ is contained in $\mathcal{T}_{\alpha}$ as an essential ideal.

We next "compute" the quotient $\mathcal{T}_{A} / \mathcal{K}\left(\mathcal{H}_{A}\right)$ :

Let $\left(A_{n}\right)_{n \in \mathbb{N}}$ be the sequence of $C^{*}$-algebras with $A_{n}=A$ for every $n \in \mathbb{N}$. For $m \geq n$ set $\varphi_{m, n}=\alpha^{m-n}: A_{n} \rightarrow A_{m}$. Let $A_{\infty}=\lim A_{n}$ be the direct limit $C^{*}$-algebra. Let $h_{n}: A \rightarrow A_{\infty}$ be the canonical map from $A=A_{n}$ to the direct limit. Define $\alpha_{\infty}: A_{\infty} \rightarrow A_{\infty}$ by setting $\alpha_{\infty}\left(h_{n}(x)\right)=h_{n}(\alpha(x))$ for $x \in A$. This is compatible with $\varphi_{m, n}$ 's and extends to $A_{\infty}$. Since $\alpha_{\infty} \circ h_{n}=$ $h_{n} \circ \alpha=h_{n} \circ \varphi_{n, n-1}=h_{n-1}$ it follows that $\alpha_{\infty}$ is an automorphism of $A_{\infty}$ (and $\alpha_{\infty}^{-1} \circ h_{n}=h_{n+1}$ ).

We set $h=h_{0}$. The algebra $A_{\infty}$ admits the following abstract characterization.

Proposition 1.2. We keep the above notation. Let $B$ be a $C^{*}$-algebra, $\sigma: A \rightarrow B$ a *homomorphism and $\beta$ an automorphism of $B$ such that $\sigma \circ \alpha=\beta \circ \sigma$. Then there exists a unique $*$-homomorphism $\sigma_{\infty}: A_{\infty} \rightarrow B$ such that $\sigma_{\infty} \circ \alpha_{\infty}=\beta \circ \sigma_{\infty}$ and $\sigma_{\infty} \circ h=\sigma$. Moreover, $A_{\infty}$ and $\alpha_{\infty}$ are uniquely determined by these conditions.

Proof. If $\sigma_{\infty}: A_{\infty} \rightarrow B$ is a $*$-homomorphism satisfying the above conditions, then $\beta^{n} \circ \sigma_{\infty} \circ h_{n}=\sigma_{\infty} \circ \alpha_{\infty}^{n} \circ h_{n}=\sigma_{\infty} \circ h=\sigma$, whence $\sigma_{\infty} \circ h_{n}=\beta^{-n} \circ \sigma$, which shows the uniqueness of $\sigma_{\infty}$.

Define $\sigma_{m}=\beta^{-m} \circ \sigma: A \rightarrow B$. If $m \geq n$, then

$$
\begin{aligned}
\sigma_{m} \circ \varphi_{m, n} & =\sigma_{m} \circ \alpha^{m-n}=\beta^{-m} \circ \sigma \circ \alpha^{m-n} \\
& =\beta^{-m} \circ \beta^{m-n} \circ \sigma=\beta^{-n} \circ \sigma=\sigma_{n} .
\end{aligned}
$$

By the universal property of direct limit there exists a $*$-homomorphism $\sigma_{\infty}: A_{\infty} \rightarrow B$. Moreover, for all $n$ we have $\sigma_{n} \circ \alpha=\beta \circ \sigma_{n}$, hence $\sigma_{\infty} \circ \alpha_{\infty}=$ $\beta \circ \sigma_{\infty}$.

Let $D$ be a $C^{*}$-algebra with an automorphism $\delta$, and let $j: A \rightarrow D$ be a *-homomorphism such that $j \circ \alpha=\delta \circ j$. Assume that if $B$ is a $C^{*}$-algebra, $\sigma: A \rightarrow B$ a $*$-homomorphism and $\beta$ an automorphism of $B$ such that $\sigma \circ \alpha=\beta \circ \sigma$, then there exists a unique $*$-homomorphism $\sigma^{\prime}: D \rightarrow B$ such that $\sigma^{\prime} \circ j=\sigma$ and $\sigma^{\prime} \circ \delta=\beta \circ \sigma^{\prime}$. Then there exist (unique) *homomorphisms $I: D \rightarrow A_{\infty}$ and $J: A_{\infty} \rightarrow D$ intertwining $\delta$ with $\alpha_{\infty}$ and such that $h \circ I=j$ and $j \circ J=h$. The uniquness statements imply that $I \circ J=\mathrm{id}_{A_{\infty}}$ and $J \circ I=\mathrm{id}_{D}$, whence $D$ is canonically $*$-isomorphic with $A_{\infty}$. 
It follows from this proposition that the construction of the pair $\left(A_{\infty}, \alpha_{\infty}\right)$ is functorial: Let $B$ be another $C^{*}$-algebra endowed with an endomorphism $\beta$. To any $*$-homomorphism $\varphi: A \rightarrow B$ such that $\varphi \circ \alpha=\beta \circ \varphi$ there corresponds a $*$-homomorphism $\varphi_{\infty}: A_{\infty} \rightarrow B_{\infty}$ such that $\varphi_{\infty} \circ \alpha_{\infty}=$ $\beta_{\infty} \circ \varphi_{\infty}$. In particular, let $\tilde{\alpha}$ be the unital endomorphism of $\tilde{A}$ extending $\alpha$. The corresponding inductive limit $C^{*}$-algebra is the algebra $\widetilde{A_{\infty}}$ obtained by adjoining a unit to $A_{\infty}$ endowed with the unital automorphism $\widetilde{\alpha_{\infty}}$ extending $\alpha_{\infty}$

In what follows, we consider $A_{\infty}$ as a $C^{*}$-subalgebra of $A_{\infty} \rtimes_{\alpha_{\infty}} \mathbb{Z}$.

Corollary 1.3. Assume that $A$ is unital and that $\alpha(1)=1$. Let $B$ be a unital $C^{*}$-algebra, $\sigma: A \rightarrow B$ a unital $*$-homomorphism and $v$ a unitary in $B$ such that $\sigma(\alpha(x))=v \sigma(x) v^{*}$, for all $x \in A$. Then there exists a unique *-homomorphism $\hat{\sigma}: A_{\infty} \rtimes_{\alpha_{\infty}} \mathbb{Z} \rightarrow B \rtimes_{\beta} \mathbb{Z}$ such that $\hat{\sigma}(d(x))=\sigma(x)$ for all $x \in A$, and $\hat{\sigma}(u)=v$ where $\beta$ is the inner automorphism of $B$ associated with $v$ and $u$ is the unitary of $A_{\infty} \rtimes_{\alpha_{\infty}} \mathbb{Z}$ defining the crossed-product.

Proof. Let $\sigma_{\infty}: A_{\infty} \rightarrow B$ be the associated $*$-homomorphism (Proposition 1.2). We have $\sigma_{\infty}\left(\alpha_{\infty}(x)\right)=v \sigma_{\infty}(x) v^{*}$, for all $x \in A_{\infty}$. By the universal property of the crossed product, there exists a unique *-homomorphism $\hat{\sigma}: A_{\infty} \rtimes_{\alpha_{\infty}} \mathbb{Z} \rightarrow B \rtimes_{\beta} \mathbb{Z}$ such that $\hat{\sigma}(x)=\sigma_{\infty}(x)$ for all $x \in A_{\infty}$ and $\hat{\sigma}(u)=v$.

Moreover, $h_{n}(x)=u^{-n} h(x) u^{n}$ for all $x \in A$ and $n \in \mathbb{N}$, so that $A_{\infty} \rtimes_{\alpha_{\infty}} \mathbb{Z}$ is generated by $h(A)$ and $u$; the uniqueness of $\hat{\sigma}$ follows immediately.

Corollary 1.4. There exists a unique *-homomorphism $\Psi: A_{\infty} \rtimes_{\alpha_{\infty}} \mathbb{Z} \rightarrow$ $\mathcal{T}_{\alpha} / \mathcal{K}\left(\mathcal{H}_{A}\right)$ such that, for all $a \in A, \Psi(h(a))$ is the image of $d(a) \in \mathcal{T}_{\alpha}$ in the quotient and, for all $x \in A_{\infty}, \Psi(u x)=v \psi(x)$, where $u$ is as above and $v$ is the image of $S^{*} \in \mathcal{M}\left(\mathcal{T}_{\alpha}\right)$ in $\mathcal{M}\left(\mathcal{T}_{\alpha} / \mathcal{K}\left(\mathcal{H}_{A}\right)\right)$.

Proof. If $A$ is unital and $\alpha(1)=1$, this is an immediate consequence of Corollary 1.3.

In the non unital case, let $\tilde{\alpha}$ be the unital endomorphism of $\tilde{A}$ extending $\alpha$. By the unital case, we get a homomorphism $\tilde{\Psi}: \widetilde{A_{\infty} \rtimes_{\alpha_{\infty}}} \mathbb{Z} \rightarrow \mathcal{T}_{\tilde{\alpha}} / \mathcal{K}\left(\mathcal{H}_{\tilde{A}}\right)$. Note that moreover $A_{\infty} \rtimes_{\alpha_{\infty}} \mathbb{Z}$ is the kernel of the map $\widetilde{A_{\infty} \rtimes_{\widetilde{\infty}}} \mathbb{Z} \rightarrow C^{*}(\mathbb{Z})$ corresponding to the unital equivariant morphism $\widetilde{A_{\infty}} \rightarrow \mathbb{C}$ and that $\mathcal{T}_{\alpha} / \mathcal{K}\left(\mathcal{H}_{A}\right)$ is the kernel of the morphism $\mathcal{T}_{\tilde{\alpha}} / \mathcal{K}\left(\mathcal{H}_{\tilde{A}}\right) \rightarrow \mathcal{T} / \mathcal{K}\left(\ell^{2}(\mathbb{N})\right)$. Since the diagram

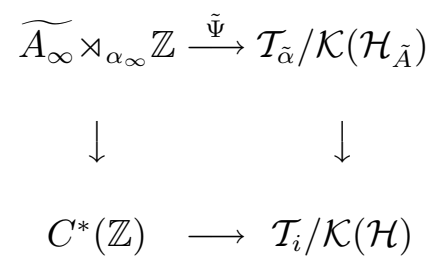


is commutative, it follows that $\tilde{\Psi}\left(A_{\infty} \rtimes_{\alpha_{\infty}}\right) \subset \mathcal{T}_{\alpha} / \mathcal{K}\left(\mathcal{H}_{A}\right)$.

Furthermore, any $\Psi: A_{\infty} \rtimes_{\alpha_{\infty}} \mathbb{Z} \rightarrow \mathcal{T}_{\alpha} / \mathcal{K}\left(\mathcal{H}_{A}\right)$ satisfying the conditions of the statement, extends to a morphism from $\widetilde{A_{\infty}} \rtimes_{\alpha_{\infty}} \mathbb{Z}$ to $\mathcal{T}_{\tilde{\alpha}} / \mathcal{K}\left(\mathcal{H}_{\tilde{A}}\right)$ mapping $u$ to $v$, from which the uniqueness of $\Psi$ follows.

Theorem 1.5. The *-homomorphism $\Psi$ of Corollary 1.4 is an isomorphism. In other words, we have an exact sequence

$$
0 \rightarrow A \otimes \mathcal{K}(\mathcal{H}) \rightarrow \mathcal{T}_{\alpha} \rightarrow A_{\infty} \rtimes_{\alpha_{\infty}} \mathbb{Z} \rightarrow 0 .
$$

Proof. For $x \in A, m, n \in \mathbb{N}$, the image of $S^{m} d(x) S^{* n}$ in $\mathcal{T}_{\alpha} / \mathcal{K}\left(\mathcal{H}_{A}\right)$ is $\Psi\left(u^{m} h(x) u^{* n}\right)$, whence $\Psi$ is onto.

To show that $\Psi$ is one to one, we may assume that $A$ is unital and $\alpha(1)=1$. Let $\left(e_{n}\right)_{n \in \mathbb{N}}$ denote the canonical basis of $\ell^{2}(\mathbb{N})$ and set $b_{n}=$ $e_{n} \otimes 1 \in \ell^{2}(\mathbb{N}, A)=\mathcal{H}_{A}$. The set of $T \in \mathcal{L}\left(\mathcal{H}_{A}\right)$ such that the sequence $\alpha_{\infty}^{-n} \circ$ $h\left(\left\langle b_{n}, T b_{n}\right\rangle\right)$ converges in norm in $A_{\infty}$ is a closed subspace of $\mathcal{L}\left(\mathcal{H}_{A}\right)$. Moreover, for all $x \in A, m, n, k \in \mathbb{N}$ we have $\alpha_{\infty}^{-k}\left(\left\langle b_{k}, S^{m} d(x) S^{* n} b_{k}\right\rangle\right)=\alpha_{\infty}^{-m} \circ h(x)$ if $k \geq m=n$ and to 0 otherwise. Consequently, $T \mapsto \lim _{n \rightarrow+\infty} \alpha_{\infty}^{-n} \circ$ $h\left(\left\langle b_{n}, T b_{n}\right\rangle\right)$ is a completely positive map $E: \mathcal{T}_{\alpha} \rightarrow A_{\infty}$, such that, for all $x \in A, m, n \in \mathbb{N}, \quad E\left(S^{m} d(x) S^{* n}\right)=0$ if $m \neq n$ and $E\left(S^{m} d(x) S^{* m}\right)=$ $\alpha_{\infty}^{-m} \circ h(x)$. Clearly $\lim _{n \rightarrow+\infty} \alpha_{\infty}^{-n} \circ h\left(\left\langle b_{n}, T b_{n}\right\rangle\right)=0$ for all $T \in \mathcal{K}\left(\mathcal{H}_{A}\right)$, so that $E$ defines a completely positive map $\Phi: \mathcal{T}_{\alpha} / \mathcal{K}\left(\mathcal{H}_{A}\right) \rightarrow A_{\infty}$. The composition $\Phi \circ \Psi$ is easily seen to be the conditional expectation $A_{\infty} \rtimes_{\alpha_{\infty}} \mathbb{Z} \mapsto A_{\infty}$ which is the identity on $A_{\infty}$ and maps $u^{k} x$ to 0 for all $x \in A_{\infty}$ and $k \neq 0$. As this conditional expectation is faithfull, $\Psi$ is one to one.

When $\alpha$ is an automorphism of $A$ we see immediately that $A_{\infty}$ identifies with $A$; therefore, the exact sequence of Theorem 1.5 is a generalization of the Toeplitz exact sequence of [10].

The following theorem characterizes the $*$-representations of the Toeplitz algebra $\mathcal{T}_{\alpha}$. If $\pi$ is a non degenerate $*$-representation of $\mathcal{T}_{\alpha}$, then $\pi \circ d$ is a $*$ representation $\sigma$ of $A$ and $T=\tilde{\pi}(S)$ is an isometry, where $\tilde{\pi}$ is the extension of $\pi$ to the multiplier algebra. For all $a \in A$ we have $\sigma(a) T=T \sigma(\alpha(a))$. The converse is also true:

Theorem 1.6. Let $B$ be a $C^{*}$-algebra and $H$ be a Hilbert $B$-module. Let $\sigma: A \rightarrow \mathcal{L}(H)$ be a *-representation of $A$ on $H$ and let $T \in \mathcal{L}(H)$ be an isometry such that $\sigma(a) T=T \sigma(\alpha(a))$. Then, there exists a *-representation $\pi: \mathcal{T}_{\alpha} \rightarrow \mathcal{L}(H)$ such that for all $x \in A, m, n \in \mathbb{N}, \pi\left(S^{m} d(x) S^{* n}\right)=$ $T^{m} \sigma(x) T^{* n}$. Moreover, $\pi$ is faithful if and only if the restriction of $\sigma$ to the kernel of $T^{*}$ is faithful.

Proof. Up to passing to $\tilde{A}$, we may assume that $A$ is a unital $C^{*}$-algebra and that $\alpha$ and $\sigma$ are unital morphisms. We first treat the case $B=\mathbb{C}$. 
Put $H_{0}=\operatorname{ker} T^{*}$ and let $H^{\prime}$ be the closure in $H$ of the union of ker $T^{* n}$ $(n \in \mathbb{N})$. As $T^{* n} \sigma(a)=\sigma\left(\alpha^{n}(a)\right) T^{* n}$, the subspaces $H_{0}$ and $H^{\prime}$ are invariant under $\sigma(A)$. Denote by $\sigma_{0}$ the restriction of $\sigma$ to $H_{0}$. Moreover, $H^{\prime}$ admits the orthogonal decomposition $H^{\prime}=\bigoplus_{n \in \mathbb{N}} T^{n} H_{0}$, therefore there exists an isomorphism of Hilbert spaces $U: \mathcal{H}_{A} \otimes_{\sigma_{0}} H_{0} \rightarrow H^{\prime}$ such that $U\left(\left(e_{n} \otimes\right.\right.$ $a) \otimes x)=T^{n} \sigma_{0}(a) x=T^{n} \sigma(a) x$ for all $n \in \mathbb{N}, a \in A, x \in H_{0}$ (where $\left(e_{n}\right)_{n \in \mathbb{N}}$ is the canonical basis of $\left.\ell^{2}(\mathbb{N})\right)$. Also $U(S \otimes 1)=T U$ and for all $a, b \in A, n \in \mathbb{N}, x \in H_{0}$

$$
\begin{aligned}
U(d(a) \otimes 1)\left(\left(e_{n} \otimes b\right) \otimes x\right) & =U\left(\left(e_{n} \otimes \alpha^{n}(a) b\right) \otimes x\right) \\
& =T^{n} \sigma\left(\alpha^{n}(a) b\right) x \\
& =\sigma(a) T^{n} \sigma(b) x \\
& =\sigma(a) U\left(\left(e_{n} \otimes b\right) \otimes x\right) .
\end{aligned}
$$

Since, the restriction of $T$ to $H^{\prime} \perp$ is a unitary operator $v$; by Corollary 1.3, there exists a $*$-representation $\pi^{\prime}: A_{\infty} \rtimes_{\alpha_{\infty}} \mathbb{Z} \mapsto \mathcal{L}\left(H^{\prime} \perp\right)$ such that $\pi^{\prime} \circ h$ is the restriction of $\sigma$ to $H^{\prime} \perp$ and $\pi^{\prime}(u)=v$. Then, the $*$-representation $\pi: x \mapsto U(x \otimes 1) U^{*}+\pi^{\prime} \circ q(x)$ satisfies the requirements of the theorem, where $q: \mathcal{T}_{\alpha} \rightarrow A_{\infty} \rtimes_{\alpha_{\infty}} \mathbb{Z}$ is the composition of the quotient map $\mathcal{T}_{\alpha} \rightarrow \mathcal{T}_{\alpha} / \mathcal{K}\left(\mathcal{H}_{A}\right)$ with $\Psi^{-1}$ of Corollary 1.4.

Now, as $\mathcal{K}\left(\mathcal{H}_{A}\right)$ is an essential ideal in $\mathcal{T}_{\alpha}$, the representation $\pi$ is faithful if and only if its restriction to $\mathcal{K}\left(\mathcal{H}_{A}\right)$ is faithful, which happens if and only if the representation $a \mapsto \pi(p \otimes a)$ is faithful, where $p \in \mathcal{K}$ is a minimal projection which, by a good choice of $p$ means that $a \mapsto \pi\left(d(a)\left(1-S S^{*}\right)\right)=$ $\sigma(a)\left(1-T T^{*}\right)$ is faithful.

We finally come to the general case $(B \neq \mathbb{C})$. We may embed $\mathcal{L}(H)$ in some $\mathcal{L}(E)$ where $E$ is a Hilbert space. Then, by the case $B=\mathbb{C}$, there exists a $*$-representation $\pi: \mathcal{T}_{\alpha} \rightarrow \mathcal{L}(E)$ whose image is obviously contained in $\mathcal{L}(H) \subset \mathcal{L}(E)$.

We end this section with a theorem showing that $\mathcal{T}_{\alpha}$ is a full corner of a crossed product. Let $C_{b}(\mathbb{Z}, A)$ be the $C^{*}$-algebra of norm bounded sequences $\left(a_{n}\right)_{n \in \mathbb{Z}}$ of elements of $A$ under pointwise operations and infinity norm. For each $p \in \mathbb{Z}$ let $j_{p}: A \rightarrow C_{b}(\mathbb{Z}, A)$ be the morphism such that $j_{p}(a)$ is the sequence whose $n^{\text {th }}$ term is zero if $n<p$ and $\alpha^{n-p}(a)$ if $n \geq p$. Let $D$ be the $C^{*}$-subalgebra of $C_{b}(\mathbb{Z}, A)$ generated by the elements $j_{p}(a)$ for $a \in A$ and $p \in \mathbb{Z}$. The shift on $C_{b}(\mathbb{Z}, A)$ induces an automorphism $\beta$ of $D$ such that $\beta \circ j_{p}=j_{p-1}$, so that $D$ is the smallest subalgebra of $C_{b}(\mathbb{Z}, A)$ containing $j_{0}(A)$ and invariant under the shift.

Lemma 1.7. The $C^{*}$-subalgebra $C_{0}(\mathbb{Z}, A)$ of $C_{b}(\mathbb{Z}, A)$ consisting of the sequences vanishing at infinity is contained in $D$ as an essential ideal. There 
is a $*$-isomorphism $\varphi: D / C_{0}(\mathbb{Z}, A) \rightarrow A_{\infty}$ such that $\varphi \circ q \circ j_{0}=h$ and $\varphi \circ q \circ \beta=\alpha_{\infty} \circ \varphi \circ q$, where $q: D \rightarrow D / C_{0}(\mathbb{Z}, A)$ is the quotient map.

Proof. For $a \in A$ and $p \in \mathbb{Z}$, the only nonzero term of the sequence $j_{p}(a)-$ $j_{p+1}(\alpha(a))$ is $a$ in $p^{t h}$ position. Consequentely $C_{0}(\mathbb{Z}, A) \subset D$ and as $D \subset$ $C_{b}(\mathbb{Z}, A)=\mathcal{M}\left(C_{0}(\mathbb{Z}, A)\right), C_{0}(\mathbb{Z}, A)$ is contained in $D$ as an essential ideal.

Note that $D$ is the inductive limit of the algebras $D_{p}=C_{0}(\mathbb{Z}, A)+$ $j_{p}(A)$. Therefore, a bounded sequence $\left(a_{n}\right)_{n \in \mathbb{Z}}$ is in $D$, if and only if, $\lim _{n \rightarrow-\infty}\left\|a_{n}\right\|=0$ and, for every $\varepsilon>0$, there exists $n \in \mathbb{Z}$ such that, for every $m \in \mathbb{N},\left\|a_{n+m}-\alpha^{m}\left(a_{n}\right)\right\| \leq \varepsilon$.

Moreover, for every $p \in \mathbb{Z}$, let $\varphi_{p}: D \rightarrow A_{\infty}$ be the map $\left(a_{n}\right)_{n \in \mathbb{Z}} \mapsto$ $\alpha_{\infty}^{-p} \circ h\left(a_{p}\right)$. Clearly $\varphi_{p} \circ j_{k}=\alpha_{\infty}^{-k}$ if $p \geq k$. Therefore, for all $x \in D$ the sequence $\varphi_{p}(x)$ converges to some element $\varphi(x)$, when $p \rightarrow+\infty$. Obviously, $\varphi$ is a $*$-homomorphism whose kernel contains $C_{0}(\mathbb{Z}, A)$ and whose image is invariant under $\alpha_{\infty}$ and contains $h(A)$; therefore $\psi$ is surjective. Let $x=\left(a_{n}\right)_{n \in \mathbb{Z}} \in \operatorname{ker} \varphi$. For every $\varepsilon$, there exists $n \in \mathbb{Z}$ such that for every $m \in \mathbb{N},\left\|a_{n+m}-\alpha^{m}\left(a_{n}\right)\right\| \leq \varepsilon$. Then

$$
\begin{aligned}
\left\|h\left(a_{n}\right)\right\| & =\left\|\alpha_{\infty}^{-n} \circ h\left(a_{n}\right)\right\| \\
& =\left\|\alpha_{\infty}^{-n} \circ h\left(a_{n}\right)-\varphi(x)\right\| \\
& =\lim _{m \rightarrow+\infty}\left\|\alpha_{\infty}^{-n-m} \circ h\left(\alpha^{m}\left(a_{n}\right)-a_{n+m}\right)\right\| \leq \varepsilon .
\end{aligned}
$$

Therefore $\lim \sup _{m \rightarrow+\infty}\left\|\alpha^{m}\left(a_{n}\right)\right\| \leq \varepsilon$, whence $\lim \sup _{m \rightarrow+\infty}\left\|a_{n+m}\right\| \leq 2 \varepsilon$. It follows that $\operatorname{ker} \varphi=C_{0}(\mathbb{Z}, A)$; therefore $\varphi$ induces the desired isomorphism.

Theorem 1.8. Let $v \in \mathcal{L}\left(\ell^{2}(\mathbb{Z}, A)\right)$ be the backward shift: i.e., $v\left(\left(x_{n}\right)_{n \in \mathbb{Z}}\right)=$ $\left(y_{n}\right)_{n \in \mathbb{Z}} \in \ell^{2}(\mathbb{Z}, A)$ where, for $y_{n}=x_{n+1}$. Moreover let $\rho: D \rightarrow \mathcal{L}\left(\ell^{2}(\mathbb{Z}, A)\right)$ be the $*$-representation such that $\rho\left(\left(a_{n}\right)_{n \in \mathbb{Z}}\right)\left(\left(x_{n}\right)_{n \in \mathbb{Z}}\right)=\left(a_{n} x_{n}\right)_{n \in \mathbb{Z}}$. The pair $(\rho, v)$ is a covariant representation of $(D, \beta)$ and the corresponding representation of $D \rtimes_{\beta} \mathbb{Z}$ is faithful. Identify $D \rtimes_{\beta} \mathbb{Z}$ with its image in $\mathcal{L}\left(\ell^{2}(\mathbb{Z}, A)\right)$; the projection $P$ of $\ell^{2}(\mathbb{Z}, A)$ onto $\ell^{2}(\mathbb{N}, A)$ is a multiplier of $D \rtimes_{\beta} \mathbb{Z}$ and $P\left(D \rtimes_{\beta} \mathbb{Z}\right) P$ is the Toeplitz algebra $\mathcal{T}_{\alpha}$; it is a full corner in $D \rtimes_{\beta} \mathbb{Z}$.

Proof. It is clear that the pair $(\rho, v)$ is a covariant representation of $(D, \beta)$. The restriction of the corresponding representation of $D \rtimes_{\beta} \mathbb{Z}$ to $C_{0}(\mathbb{Z}, A) \rtimes_{\beta} \mathbb{Z}$ is the canonical isomorphism of $C_{0}(\mathbb{Z}, A) \rtimes_{\beta} \mathbb{Z}$ with the algebra of compact operators in $\ell^{2}(\mathbb{Z}, A)$. As $C_{0}(\mathbb{Z}, A)$ is an essential ideal in $D, C_{0}(\mathbb{Z}, A) \rtimes_{\beta} \mathbb{Z}$ is an essential ideal in $D \rtimes_{\beta} \mathbb{Z}$ therefore the representation of $D \rtimes_{\beta} \mathbb{Z}$ associated with $(\rho, v)$ is faithful.

As $P$ is a multiplier of $\rho(D)$, it is a multiplier of $D \rtimes_{\beta} \mathbb{Z}$. Moreover, $(1-$ $P) \rho(D) \subset \mathcal{K}\left(\ell^{2}(\mathbb{Z}, A)\right)$ so that $(1-P)(D \rtimes \mathbb{Z}) \subset \mathcal{K}\left(\ell^{2}(\mathbb{Z}, A)\right)$; it follows that 
$D \rtimes \mathbb{Z}=\mathcal{T}_{\alpha}+\mathcal{K}\left(\ell^{2}(\mathbb{Z}, A)\right)$; as $\mathcal{K}\left(\ell^{2}(\mathbb{N}, A)\right) \subset P\left(D \rtimes_{\beta} \mathbb{Z}\right) P$ and $\mathcal{K}\left(\ell^{2}(\mathbb{N}, A)\right)$ is a full corner in $\mathcal{K}\left(\ell^{2}(\mathbb{Z}, A)\right)$, it follows that $P\left(D \rtimes_{\beta} \mathbb{Z}\right) P$ is a full corner in $D \rtimes_{\beta} \mathbb{Z}$.

Now for all $m, n \in \mathbb{N}$, and $a \in A$, we have $v^{* m} \rho \circ j_{0}(a) v^{n}=P\left(v^{* m} \rho \circ\right.$ $\left.j_{0}(a) v^{n}\right) P$ and acts on $\ell^{2}(\mathbb{N}, A)$ as $S^{m} d(a) S^{* n}$. It follows that $P\left(D \rtimes_{\beta} \mathbb{Z}\right) P$ contains $\mathcal{T}_{\alpha}$. Now $D \rtimes_{\beta} \mathbb{Z}$ is generated by $v^{k} \rho \circ j_{p}(a)$ where $p, k \in \mathbb{Z}, a \in$ A. Moreover, if $n \in \mathbb{N}, \rho\left(j_{p-n}(a)-j_{p}\left(\alpha^{n}(a)\right)\right) \in \mathcal{K}\left(\ell^{2}(\mathbb{N}, A)\right) \subset \mathcal{T}_{\alpha}$; it is enough to show that $P\left(v^{k} \rho \circ j_{p}(a)\right) P \in \mathcal{T}_{\alpha}$ when $p \geq 0$ and $p-k \geq 0$. But $v^{k} \rho \circ j_{p}(a)=v^{k-p} \rho \circ j_{0}(a) v^{p}$ and the result follows.

\section{2. $K K$-Groups.}

In $([\mathbf{1 0}])$ it is proved that, when $\alpha$ is an automorphism, the canonical inclusion of $A$ in $\mathcal{T}_{\alpha}$ induces an isomorphism at the $K$-theory level, and deduced a six term exact sequence computing the $K$-groups of a crossed-product by $\mathbb{Z}$. Here we prove that this holds in general, by showing that the same map considered as an element of the group $K K\left(A, \mathcal{T}_{\alpha}\right)$ is invertible. As a consequence of this fact, we obtain a generalized version of Pimsner-Voiculescu exact sequence for endomorphisms.

Recall (cf. [6]) that if $A$ and $B$ are $C^{*}$-algebras, an element of $K K(A, B)$ is given by the homotopy class of a triple $(\mathcal{E}, \pi, F)$, where $\mathcal{E}$ is a $\mathbb{Z} / 2 \mathbb{Z}$ graded Hilbert $B$-module, $\pi: A \rightarrow \mathcal{L}(\mathcal{E})$ is a $*$-representation of $A$ on $\mathcal{L}(\mathcal{E})$ as degree zero operators, and $F \in \mathcal{L}(\mathcal{E})$ has degree 1 such that for all $a \in A$, $[\pi(a), F] \in \mathcal{K}(\mathcal{E}), \pi(a)\left(F-F^{*}\right) \in \mathcal{K}(\mathcal{E})$ and $\pi(a)\left(1-F^{2}\right) \in \mathcal{K}(\mathcal{E})$.

Given a $*$-homomorphism $\varphi: A \rightarrow B$ we denote by $[\varphi]$ the element of $K K(A, B)$ given by the class of $(B, \varphi, 0)$.

We keep the notation of the first section. In particular $d: A \rightarrow \mathcal{T}_{\alpha}$ is the embedding of $A$ into $\mathcal{T}_{\alpha}$. Set $\mathcal{E}^{(0)}=\ell^{2}(\mathbb{N}, A)$ and let $\mathcal{E}^{(1)}=\ell^{2}(\mathbb{N} \backslash\{0\}, A)$ be the subspace of $\mathcal{E}^{(0)}$ with zero in the first coordinate. Let $Q: \mathcal{E}^{(0)} \rightarrow \mathcal{E}^{(1)}$ be the orthogonal projection. Let $\mathcal{E}$ denote the $\mathbb{Z} / 2 \mathbb{Z}$-graded Hilbert $A$-module $\mathcal{E}^{(0)} \oplus \mathcal{E}^{(1)}$.

By Theorem 1.6, there is a $*$-representation of $\pi^{-}: \mathcal{T}_{\tilde{\alpha}} \rightarrow \mathcal{L}\left(\mathcal{E}^{(1)}\right)$ such that $\pi^{-} \circ d$ is the restriction of $d$ to the invariant subspace $\mathcal{E}^{(1)}$ of $\mathcal{E}^{(0)}$ and $\pi^{-}(S)=Q S Q=S Q$. In fact $\pi^{-}(x)=S \tau_{\alpha}(x) S^{*}$ where $\tau_{\alpha}: \mathcal{T}_{\alpha} \rightarrow \mathcal{T}_{\alpha}$ is the map induced by $\alpha: A \rightarrow A$. Let $\pi: \mathcal{T}_{\alpha} \rightarrow \mathcal{L}(\mathcal{E})$ be the $*$-representation such that for $x \in \mathcal{T}_{\alpha}, \xi \in \mathcal{E}^{(0)} \eta \in \mathcal{E}^{(1)}$ we have $\pi(x)(\xi, \eta)=\left(x \xi, \pi^{-}(x) \eta\right)$. Let $F \in \mathcal{L}(\mathcal{E})$ be defined for $\xi \in \mathcal{E}^{(0)} \eta \in \mathcal{E}^{(1)}$ by $F(\xi, \eta)=(\eta, Q \xi)$.

Lemma 2.1. The triple $(\mathcal{E}, \pi, F)$ defines an element of $K K\left(\mathcal{T}_{\alpha}, A\right)$.

Proof. Clearly $F=F^{*}$ and $1-F^{2}$ is the projection $(\xi, \eta) \mapsto((1-Q) \xi, \eta)$, so that $\left(1-F^{2}\right) \mathcal{T}_{\alpha} \subset \mathcal{K}(\mathcal{E})$. If $a \in A$, then $\pi \circ d(a)$ and $F$ commute. Moreover 
$(F \pi(S)-\pi(S) F)(\xi, \eta)=(S \eta-S \eta, Q S \xi-S Q \xi)=(0, S(1-Q) \xi)$, so that $(F \pi(S)-\pi(S) F) \mathcal{T}_{\alpha} \subset \mathcal{K}(\mathcal{E})$.

Definition 2.2. We denote by $[d]$ the class of the morphism $d$ in $K K\left(A, \mathcal{T}_{\alpha}\right)$ and by $\beta$ the class of the triple $(\mathcal{E}, \pi, F)$ in $K K\left(\mathcal{T}_{\alpha}, A\right)$.

Theorem 2.3. We have $[d] \otimes_{\mathcal{T} \alpha} \beta=1_{A} \in K K(A, A)$ and $\beta \otimes_{A}[d]=1_{\mathcal{T}_{\alpha}} \in$ $K K\left(\mathcal{T}_{\alpha}, \mathcal{T}_{\alpha}\right)$. In particular, the $C^{*}$-algebras $A$ and $\mathcal{T}_{\alpha}$ are $K K$-equivalent.

Proof. Here the Kasparov products are easily computed: We have $[d] \otimes_{\mathcal{T} \alpha} \beta=$ $d^{*}(\beta)$ and $\beta \otimes_{A}[d]=d_{*}(\beta)$. Since $\pi \circ d$ commutes with $F$ and $F$ is a self adjoint partial isometry it follows that the class of $(\mathcal{E}, \pi \circ d, F)$ coincides with the class of $\left(\left(1-F^{2}\right) \mathcal{E}, i, 0\right)$ where $i$ is the restriction of $\pi \circ d$ to $\left(1-F^{2}\right) \mathcal{E}=A$ and is therefore given by the identity map $A \rightarrow A=\mathcal{K}(A)$, hence $d^{*}(\beta)=1_{A}$.

Now $d_{*}(\beta)$ is given by $(\mathcal{F}, \sigma, G)$ where $\mathcal{F}=\mathcal{E} \otimes_{A} \mathcal{T}_{\alpha}, G=F \otimes 1$ and, for all $x \in \mathcal{T}_{\alpha}, \quad \sigma(x)=\pi(x) \otimes 1$. Therefore $\mathcal{F}^{(0)}=\ell^{2}\left(\mathbb{N}, \mathcal{T}_{\alpha}\right), \quad \mathcal{F}^{(1)}=$ $\ell^{2}\left(\mathbb{N} \backslash\{0\}, \mathcal{T}_{\alpha}\right), \sigma=\sigma^{(0)} \oplus \sigma^{(1)}$ where $\sigma^{(0)}: \mathcal{T}_{\alpha} \rightarrow \mathcal{L}\left(\ell^{2}\left(\mathbb{N}, \mathcal{T}_{\alpha}\right)\right)$ and $\sigma^{(1)}:$ $\mathcal{T}_{\alpha} \rightarrow \mathcal{L}\left(\ell^{2}\left(\mathbb{N} \backslash\{0\}, \mathcal{T}_{\alpha}\right)\right)$ are defined by $\sigma^{(i)}(d(a)) \xi(n)=d\left(\alpha^{n}(a)\right) \xi(n)$ for $a \in A$ and $\sigma^{(i)}(S) \xi(n)=\xi(n-1)$ if $n>i$ and $\sigma^{(i)}(S) \xi(i)=0(i=0,1)$.

For each $t \in\left[0, \frac{\pi}{2}\right]$ let $T_{t} \in \mathcal{L}\left(\mathcal{F}^{(0)}\right)$ be defined by

$$
\left(T_{t} \xi\right)(n)= \begin{cases}\xi_{n-1} & \text { if } n \geq 2 \\ (\cos t) \xi_{0} & \text { if } n=1 \\ (\sin t) S \xi_{0} & \text { if } n=0\end{cases}
$$

Then,

$$
\left(T_{t}^{*} \xi\right)(n)= \begin{cases}\xi_{n+1} & \text { if } n \geq 1 \\ (\cos t) \xi_{1}+(\sin t) S^{*} \xi_{0} & \text { if } n=0\end{cases}
$$

One checks immediately that $T_{t}$ is an isometry such that $\sigma^{(0)}(d(a)) T_{t}=$ $T_{t} \sigma^{(0)}(d(\alpha(a)))$ for every $a \in A$. Hence, by Theorem 1.6, there exists a $*-$ representation $\sigma_{t}^{(0)}: \mathcal{T}_{\alpha} \rightarrow \mathcal{L}\left(\mathcal{F}^{(0)}\right)$ defined by $\sigma_{t}(S)=T_{t}$ and $\sigma_{t}^{(0)}(d(a))=$ $\sigma^{(0)}(d(a))$. Moreover, for every $x \in \mathcal{T}_{\alpha}, \sigma_{t}^{(0)}(x)-\sigma^{(0)} \in \mathcal{K}\left(\mathcal{F}^{(0)}\right)$. Consequentely, $\left(\mathcal{F}, \sigma_{t}^{(0)} \oplus \sigma^{(1)}, G\right)$ is a homotopy connecting the elements $d_{*}(\beta)$ and $\left(\mathcal{F}, \sigma_{\pi / 2}^{(0)} \oplus \sigma^{(1)}, G\right)$.

Now $\mathcal{F}^{(0)}$ admits the decomposition $\mathcal{F}^{(0)}=\mathcal{T}_{\alpha} \oplus \mathcal{F}^{(1)}$ which is invariant under $\sigma_{\pi / 2}^{(0)}$. It follows that $\left(\mathcal{F}, \sigma_{\pi / 2}^{(0)} \oplus \sigma^{(1)}, G\right)$ is the sum of $1_{\mathcal{T}_{\alpha}}$ and a degenerate element. We conclude that $d_{*}(\beta)=1_{\mathcal{T}_{\alpha}}$. 
Lemma 2.4. Let $\theta: A \rightarrow \mathcal{T}_{\alpha}$ be defined by $\theta(a)=d(a)\left(1-S S^{*}\right)$. Then, $[\theta] \otimes_{\mathcal{T}_{\alpha}} \beta=1_{A}-[\alpha] \in K K(A, A)$.

Proof. The element $[\theta] \otimes_{\mathcal{T} \alpha} \beta=\theta^{*}(\beta)$ is defined by $(\mathcal{E}, \pi \circ \theta, F)$. Given $\xi \in$ $\mathcal{E}^{(0)}=\ell^{2}(\mathbb{N}, A)$ we have $(\pi \circ \theta(a) \xi)(n)=\pi\left(d(a)\left(1-S S^{*}\right)\right) \xi(n)=0$ if $n \neq 0$ and $(\pi \circ \theta(a) \xi)(0)=a \xi(0)$. On the other hand, if $\xi \in \mathcal{E}^{(1)}=\ell^{2}(\mathbb{N} \backslash\{0\}, A)$, then $(\pi \circ \theta(a) \xi)(n)=\pi\left(d(a)\left(1-S S^{*}\right)\right) \xi(n)=0$ if $n \neq 1$ and $(\pi \circ \theta(a) \xi)(1)=$ $\alpha(a) \xi(1)$. Hence, up to a degenerate module $\theta^{*}(\beta)$ is represented by the triple $\left(\mathcal{E}^{\prime}, \mu, 0\right)$ where $\mathcal{E}^{\prime(0)}=\mathcal{E}^{\prime}(1)=A$ and, for $a \in A, \mu(a)$ is given by the matrix $=\left(\begin{array}{cc}a & \sigma \\ 0 & \alpha(a)\end{array}\right)$.

Using exactness of Connes-Higson's E-theory ([2]), Theorem 2.3 to replace $\mathcal{T}_{\alpha}$ by $A$ in the exact sequence of $E$-groups associated with the extension of $C^{*}$-algebras of Theorem 1.5 and Lemma 2.4 to compute the map from $E(D, A)$ (resp. $E(A, D))$ into itself, we get:

Theorem 2.5. Let $A, \alpha$, and $\alpha_{\infty}$ be as in 1.6. Then we have exact sequences of Connes-Higson's E-groups

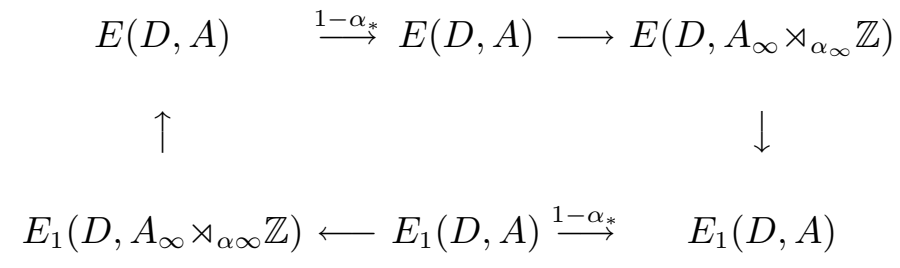

and

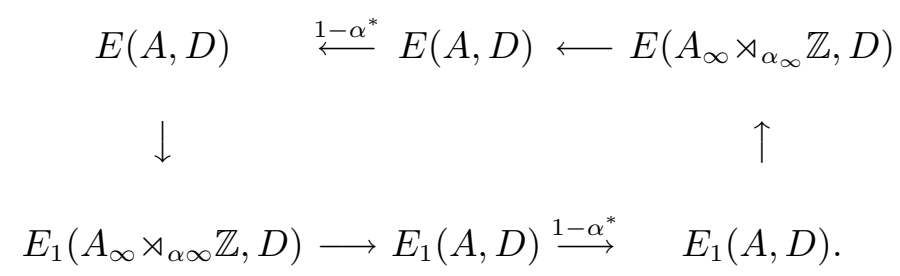

Remarks 2.6.

(a) When $\alpha$ is an automorphism, we recover Pimsner-Voiculescu's exact sequences $([\mathbf{1 0}])$.

(b) The same result holds of course with the " $K K^{\text {nuc" }}$-groups of [11] instead of $E$-groups.

(c) We may compare the two Toeplitz extensions comming from $\alpha$ and $\alpha_{\infty}$. We get a diagram of the form: 


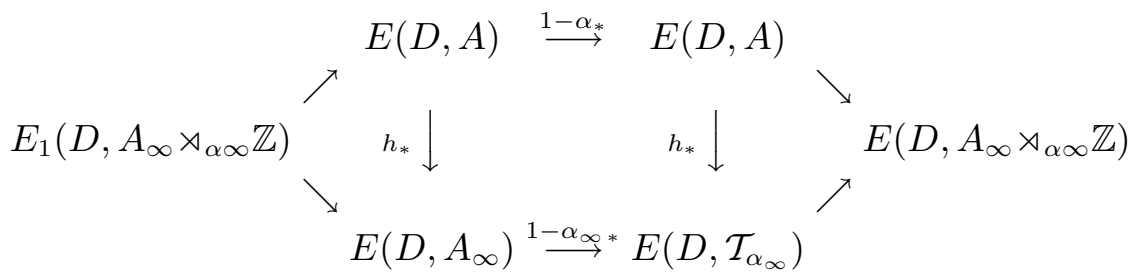

for which both top and bottom lines are exact. It follows in particular that $h_{*}$ induces an isomorphism from the kernel of $1-\alpha_{*}$ onto the kernel of $1-\alpha_{\infty *}$ and from the cokernel of $1-\alpha_{*}$ onto the cokernel of $1-\alpha_{\infty *}$. Note that when $D=\mathbb{C}$, the group $E\left(D, A_{\infty}\right)=K_{0}\left(A_{\infty}\right)$ is the inductive limit of $\left(K_{0}(A), \alpha_{*}\right)$ and it is clear that $h_{*}$ induces isomorphisms at these kernel and cokernel levels.

\section{Semigroup of Endomorphisms.}

In this section we define the Topelitz algebra associated with a semigroup of endomorphisms of a $C^{*}$-algebra $A$ and formulate the corresponding Toeplitz extension.

By a semigroup of endomorphisms of a $C^{*}$-algebra $A$ we mean a morphism $\alpha: t \mapsto \alpha^{t}$ from the (additive) monoid $\mathbb{R}_{+}$to the monoid $\operatorname{End}(A)$ of endomorphisms of a $A$ satisfying $\alpha^{0}=i d_{A}$ and $t \mapsto \alpha^{t}(a)$ is continuous for every $a \in A$. As $\alpha$, is a morphism for all $s, t \in \mathbb{R}_{+}$, we have $\alpha^{t+s}=\alpha^{t} \circ \alpha^{s}$.

Note that we have:

Lemma 3.1. Let $\left(\alpha^{t}\right)_{t \in \mathbb{R}_{+}}$be a semigroup of endomorphism of a $C^{*}$-algebra $A$. If $\alpha^{t}$ is an automorphism of $A$ for some $t>0$, then $\alpha^{s} \in \operatorname{Aut}(A)$ for every $s \in \mathbb{R}_{+}$.

The continuous analogue of the Toeplitz algebra of Section 1 is defined as follows.

Let $\pi_{\alpha}: A \rightarrow \mathcal{L}\left(L^{2}\left(\mathbb{R}_{+}\right) \otimes A\right)$ be defined by $\pi_{\alpha}(a) \xi(t)=\alpha^{t}(a) \xi(t)$ for every $\xi \in L^{2}\left(\mathbb{R}_{+}\right) \otimes A=L^{2}\left(\mathbb{R}_{+}, A\right)$ and every $a \in A$. Let $S_{t} \in \mathcal{L}\left(L^{2}\left(\mathbb{R}_{+}\right) \otimes A\right)$ be defined by $\left(S_{t} \xi\right)(s)=\xi(s-t)$ if $s \geq t$ and $\left(S_{t} \xi\right)(s)=0$ if $s<t$.

Clearly $\left(S_{t}\right)_{t \in \mathbb{R}_{+}}$is a semigroup of isometries of $\mathcal{L}\left(L^{2}\left(\mathbb{R}_{+}\right)\right)$. Moreover, for every $a \in A$ and every $t \in \mathbb{R}_{+}$we have $\pi_{\alpha}(a) S_{t}=S_{t} \pi_{\alpha}\left(\alpha^{t}(a)\right)$.

It follows that the integrals $\int_{0}^{\infty} \int_{0}^{\infty} S_{s} \pi_{\alpha}(a(s, t)) S_{t}^{*} d s d t$ where $(s, t) \mapsto a(s, t)$ is a continuous function from $\mathbb{R}_{+} \times \mathbb{R}_{+}$to $A$ with compact support form a *-subalgebra of $\mathcal{L}\left(L^{2}\left(\mathbb{R}_{+}\right) \otimes A\right)$.

Definition 3.2. Let $A$ and $\alpha$ be as above. The associated Toeplitz algebra, denoted by $\mathcal{T}_{\alpha}$, is the closure in $\mathcal{L}\left(L^{2}\left(\mathbb{R}_{+}\right) \otimes A\right)$ of the algebra formed by the integrals

$$
\int_{0}^{\infty} \int_{0}^{\infty} S_{s} \pi_{\alpha}(a(s, t)) S_{t}^{*} d s d t
$$


where $(s, t) \mapsto a(s, t)$ from $\mathbb{R}_{+} \times \mathbb{R}_{+}$to $A$ is continuous with compact support.

\section{Remarks.}

(a) By density of continuous functions with compact support in $L^{1}$-functions, for every $a \in L^{1}\left(\mathbb{R}_{+} \times \mathbb{R}_{+} ; A\right), \int_{0}^{\infty} \int_{0}^{\infty} S_{s}^{*} \pi_{\alpha}(a(s, t)) S_{t} d s d t \in \mathcal{T}_{\alpha}$.

(b) Let $b: t \mapsto b(t)$ be a continuous function from $\mathbb{R}_{+}$to $A$ with compact support; for $s, t \in \mathbb{R}^{+}$set $a(s, t)=\alpha^{t}(b(s-t))$ when $t \leq \inf (1, s)$ and $a(s, t)=0$ otherwise. Then

$$
\begin{aligned}
\int_{0}^{\infty} \int_{0}^{\infty} S_{s} \pi_{\alpha}(a(s, t)) S_{t}^{*} d s d t & =\int_{0}^{1} d t\left(\int_{s}^{\infty} S_{s} S_{t}^{*} \pi_{\alpha}(b(s-t)) d s\right) \\
& =\int_{0}^{\infty} S_{s} \pi_{\alpha}(b(s)) d s
\end{aligned}
$$

It follows that $\int_{0}^{\infty} S_{s} \pi_{\alpha}(b(s)) d s \in \mathcal{T}_{\alpha}$. Clearly $\mathcal{T}_{\alpha}$ is the $C^{*}$-subalgebra of $\mathcal{L}\left(L^{2}\left(\mathbb{R}_{+}\right) \otimes A\right)$ generated by these elements.

As in the case of a single endomorphism we have:

Proposition 3.3. The Toeplitz algebra $\mathcal{T}_{\alpha}$ contains the ideal of compact operators of $L^{2}\left(\mathbb{R}_{+}\right) \otimes A$.

Proof. Set $V=1-2 \int_{0}^{\infty} e^{-t} S_{t} d t$. It is an isometry and the kernel of $V^{* n}$ is formed by the functions $t \mapsto e^{-t} P$ where $P$ is a polynomial of degree less than $n$. It follows that $\mathcal{T}_{\alpha}$ contains the elements $k \pi_{\alpha}(a) k^{\prime}$ for every $k, k^{\prime} \in$ $\mathcal{K}\left(L^{2}\left(\mathbb{R}_{+}\right)\right)$and $a \in A$. Let $k \in \mathcal{K}\left(L^{2}\left(\mathbb{R}_{+}\right)\right)$and $a \in A$; by continuity of the mapping $t \mapsto \alpha^{t}(a)$ given $\varepsilon>0$ there exists $\eta>0$ such that $\left\|\alpha^{t}(a)-a\right\|<\varepsilon$ whenever $t \leq \eta$. But we can choose $x, y \in \mathcal{K}\left(L^{2}\left(\mathbb{R}_{+}\right)\right)$such that $k=x y$ and $x$ has support in $[0, \eta]$. It follows that $\left\|x \pi_{\alpha}(a) y-k \otimes a\right\|<\varepsilon$, whence $k \otimes a \in \mathcal{T}_{\alpha}$.

Next we show that $\mathcal{T}_{\alpha}$ is a full corner of an appropriate crossed product.

Let $A_{\infty}$ be the $C^{*}$-algebra as defined in section 1 corresponding to the endomorphism $\alpha^{1}$ of $A$, and let $h: A \rightarrow A_{\infty}$ be the canonical map. Then $\alpha^{1}$ induces an automorphism on $A_{\infty}$ which we denote by $\alpha_{\infty}^{1}$. Since $\alpha^{1} \circ \alpha^{t}=$ $\alpha^{t} \circ \alpha^{1}$ each $\alpha^{t}$ induces an endomorphism, $\alpha_{\infty}^{t}$ of the algebra $A_{\infty}$. Hence, by Lemma 3.1 we obtain an action of $\mathbb{R}$ on $A_{\infty}$ corresponding to the family $\left(\alpha_{\infty}^{t}\right)_{t \in \mathbb{R}_{+}}$which will be denoted by $\alpha_{\infty}$.

Let $C_{b}(\mathbb{R}, A)$ be the $C^{*}$-algebra of bounded functions from $\mathbb{R}$ to $A$. Let $D \subset C_{b}(\mathbb{R} ; A)$ be the subalgebra of elements $a \in C_{b}(\mathbb{R}, A)$ such that $\lim _{t \rightarrow-\infty}\|a(t)\|=0$, and for every $\varepsilon>0$, there exists $t \in \mathbb{R}$ such that for every $s>0,\left\|a(s+t)-\alpha^{s}(a(t))\right\| \leq \varepsilon$. Let $\beta: \mathbb{R} \rightarrow$ Aut $(D)$ be defined by $\left(\beta^{t} f\right)(s)=f(s-t)$. Clearly $\mathrm{D}$ contains $C_{\circ}(\mathbb{R}, D)$ as an ideal. 
Lemma 3.4. There exists a *-isomorphism $\varphi: D / C_{0}(\mathbb{R}, A) \rightarrow A_{\infty}$ such that for every $a \in D$ we have $\varphi \circ q(a)=\lim _{t \rightarrow+\infty} \alpha_{\infty}^{-t} \circ h(a(t))$, where $q$ : $D \rightarrow D / C_{0}(\mathbb{R}, A)$ is the quotient map.

Proof. It is easy to see that for every $a \in D$, the function $t \mapsto \alpha_{\infty}^{-t} \circ h(a(t))$ admits a limit when $t \rightarrow+\infty$. It follows that $\varphi$ is well defined on the quotient. For each $a \in A$ let $\hat{a}(t)=\alpha^{t}(a)$. Then, $\hat{a} \in D$ and $\lim _{t \rightarrow+\infty} \alpha_{\infty}^{-t}$ 。 $h(\hat{a}(t))=h(a)$. It follows that $\varphi$ is surjective.

Moreover, if $a \in \operatorname{ker} \varphi \circ q$, for every $\varepsilon$, there exists $t \in \mathbb{R}$ such that for every $s \in \mathbb{R}_{+},\left\|a(s+t)-\alpha^{s}(a(t))\right\| \leq \varepsilon$. Choose $t$ such that $\|h(a(t))\|=$ $\left\|\alpha_{\infty}^{-t} \circ h(a(t))-\varphi \circ h(a)\right\| \leq \varepsilon$. Therefore lim $\sup _{s \rightarrow+\infty}\left\|\alpha^{s}(a(t))\right\| \leq \varepsilon$, whence $\lim \sup _{s \rightarrow+\infty}\|a(s+t)\| \leq 2 \varepsilon$. It follows that $\operatorname{ker} \varphi \circ q=C_{0}(\mathbb{R}, A)$. Hence $\varphi$ is an isomorphism.

Theorem 3.5. Let $v_{t} \in \mathcal{L}\left(L^{2}(\mathbb{R}, A)\right)$ be defined by $\left(v_{t} \xi\right)(s)=\xi(s+t)$. Moreover let $\rho: D \rightarrow \mathcal{L}\left(L^{2}(\mathbb{R}, A)\right)$ be the *-representation such that $(\rho(a) \xi)(s)=$ $a_{s} \xi_{s}$. The pair $(\rho, v)$ is a covariant representation of $(D, \beta)$ and the corresponding representation of $D \rtimes_{\beta} \mathbb{R}$ is faithful. Identify $D \rtimes_{\beta} \mathbb{R}$ with its image in $\mathcal{L}\left(L^{2}(\mathbb{R}, A)\right)$; the projection $P$ of $L^{2}(\mathbb{R}, A)$ onto $L^{2}\left(\mathbb{R}_{+}, A\right)$ is a multiplier of $D \rtimes_{\beta} \mathbb{Z}$ and $P\left(D \rtimes_{\beta} \mathbb{R}\right) P$ is the Toeplitz algebra $\mathcal{T}_{\alpha}$; it is a full corner in $D \rtimes_{\beta} \mathbb{R}$.

Proof. It is clear that the pair $(\rho, v)$ is a covariant representation of $(D, \beta)$. The restriction of the corresponding representation of $D \rtimes_{\beta} \mathbb{R}$ to $C_{0}(\mathbb{R}, A) \rtimes_{\beta} \mathbb{R}$ is the canonical isomorphism of $C_{0}(\mathbb{R}, A) \rtimes_{\beta} \mathbb{R}$ with the algebra of compact operators in $L^{2}(\mathbb{R}, A)$. As $C_{0}(\mathbb{R}, A)$ is an essential ideal in $D, C_{0}(\mathbb{R}, A) \rtimes_{\beta} \mathbb{R}$ is an essential ideal in $D \rtimes_{\beta} \mathbb{R}$ therefore the representation of $D \rtimes_{\beta} \mathbb{R}$ associated with $(\rho, v)$ is faithful.

Let $f$ be a continuous function on $R$ such that $f(t)=1$ if $t<0$ and $f(t)=0$ if $t>1$. As $f$ is a multiplier of $D$ and $f D \subset C_{0}(\mathbb{R}, A), f$ defines a multiplier of $D \rtimes_{\beta} \mathbb{R}$ and $f D \rtimes_{\beta} \mathbb{R} \subset \mathcal{K}\left(L^{2}(\mathbb{R}, A)\right)$. As $(1-P)$ is a multiplier of $\mathcal{K}\left(L^{2}(\mathbb{R}, A)\right)$ and $(1-P)=(1-P) f$, it follows that $P$ is a multiplier of $D \rtimes_{\beta} \mathbb{R}$ and $(1-P) D \rtimes_{\beta} \mathbb{R} \subset \mathcal{K}\left(L^{2}(\mathbb{R}, A)\right)$. As $\mathcal{K}\left(L^{2}\left(\mathbb{R}_{+}, A\right)\right) \subset P\left(D \rtimes_{\beta} \mathbb{R}\right) P$, it follows that $P\left(D \rtimes_{\beta} \mathbb{R}\right) P$ is a full corner in $D \rtimes_{\beta} \mathbb{R}$.

Let $D_{0} \subset D$ be the set of $b \in D$ such that for all $u \geq 0, b(u)=\alpha^{u}(b(0))$. Let $(s, t) \mapsto a(s, t)$ be a continuous function from $\mathbb{R}_{+} \times \mathbb{R}_{+}$to $A$ with compact support. Let $b: \mathbb{R}_{+} \times \mathbb{R}_{+} \rightarrow D_{0}$ be a function such that for every $s, t \in$ $\mathbb{R}_{+}, b(s, t)(0)=a(s, t)$. Then

$$
\int_{0}^{\infty} \int_{0}^{\infty} v_{s}^{*} P b(s, t) v_{t} d s d t \in P\left(D \rtimes_{\beta} \mathbb{R}\right) P
$$


and acts on $L^{2}\left(\mathbb{R}_{+}, A\right)$ as

$$
\int_{0}^{\infty} \int_{0}^{\infty} S_{s} \pi_{\alpha}(a(s, t)) S_{t}^{*} d s d t
$$

It follows that $P\left(D \rtimes_{\beta} \mathbb{R}\right) P$ contains $\mathcal{T}_{\alpha}$.

Now $D \rtimes_{\beta} \mathbb{R}$ is generated by integrals over $s, t$ of terms of the form $v_{s} \beta^{t}(a(s, t))=v_{s-t} a(s, t) v_{t}$, where for $s, t \in \mathbb{R}, a(s, t) \in D_{0}$. Moreover, since $\cup \beta_{t}\left(D_{\circ}\right)$ is dense in $D$ and $\beta_{t}\left(D_{0}\right)$ increases with $t$, we may assume $t>0$ and $t \geq s$. Moreover $\int_{0}^{\infty} \int_{-\infty}^{t} v_{s-t}(1-P) b(s, t) v_{t} d s d t \in \mathcal{K}\left(L^{2}(\mathbb{R}, A)\right)$ and hence $P\left(\int_{0}^{\infty} \int_{-\infty}^{t} v_{s-t} b(s, t) v_{t} d s d t\right) P$ is the sum of

$$
P\left(\int_{0}^{\infty} \int_{-\infty}^{t} v_{s-t}(1-P) b(s, t) v_{t} d s d t\right) P \in \mathcal{K}\left(L^{2}\left(\mathbb{R}_{+}, A\right)\right)
$$

and

$$
\int_{0}^{\infty} \int_{-\infty}^{t} v_{s-t} P b(s, t) v_{t} d s d t \in \mathcal{T}_{\alpha}
$$

and the result follows.

Remark. Note that any isomorphism of $L^{2}(\mathbb{R}, A)$ with $L^{2}\left(\mathbb{R}_{+}, A\right)$ which is the identity on $L^{2}((k,+\infty) ; A)$ (for $k$ large enough) obviously induces an isomorphism between $D \rtimes_{\beta} \mathbb{R}$ and $\mathcal{T}_{\alpha}$.

Corollary 3.6. The quotient algebra $\mathcal{T}_{\alpha} / \mathcal{K}\left(L^{2}\left(\mathbb{R}_{+}, A\right)\right)$ is naturally isomorphic with $A_{\infty} \rtimes_{\alpha \infty} \mathbb{R}$. In other words, there is an exact sequence

$$
0 \rightarrow \mathcal{K}\left(L^{2}\left(\mathbb{R}_{+}, A\right)\right) \rightarrow \mathcal{T}_{\alpha} \rightarrow A_{\infty} \rtimes_{\alpha \infty} \mathbb{R} \rightarrow 0 .
$$

Proof. By Theorem 3.5, since $(1-P) D \rtimes_{\beta} \mathbb{R}$ is contained in $\mathcal{K}\left(L^{2}(\mathbb{R}, A)\right)$ it follows that $\mathcal{T}_{\alpha}+\mathcal{K}\left(L^{2}(\mathbb{R}, A)\right)=D \rtimes_{\beta} \mathbb{R}$. Hence, $\mathcal{T}_{\alpha} / \mathcal{K}\left(L^{2}\left(\mathbb{R}_{+}, A\right)\right)$ is canonically isomorphic to $D \rtimes_{\beta} \mathbb{R} / \mathcal{K}\left(L^{2}(\mathbb{R}, A)\right)=D \rtimes_{\beta} \mathbb{R} / C_{0}(\mathbb{R}, A) \rtimes_{\beta} \mathbb{R}$; it is therefore isomorphic to $\left(D / C_{0}(\mathbb{R}, A)\right) \rtimes_{\alpha_{\infty}} \mathbb{R}$, i.e., to $A_{\infty} \rtimes_{\alpha \infty} \mathbb{R}$ (see Lemma $3.4)$.

Let us now come to $K$-theoretic considerations.

Theorem 3.7. The morphism $h: A \rightarrow A_{\infty}$ is an isomorphism in Etheory. The $C^{*}$-algebras $D$ and $\mathcal{T}_{\alpha}$ are contractible in E-theory, i.e., for any $C^{*}$-algebra $B$ the groups $E\left(\mathcal{T}_{\alpha}, B\right), E(D, B), E\left(B, \mathcal{T}_{\alpha}\right)$ and $E(B, D)$ are trivial.

Proof. Set $D_{+}=D / C_{0}((-\infty, 0), A)$. The exact sequence $0 \rightarrow C_{0}\left(\mathbb{R}_{+}, A\right) \rightarrow$ $D_{+} \rightarrow A_{\infty} \rightarrow 0$ is an asymptotic morphism $\varphi$ from $A_{\infty}$ to $A$. 
Note that for every $C^{*}$-algebra $B$, the identity element of the ring $E(B, B)$ is given by the asymptotic morphism associated with the exact sequence $0 \rightarrow C_{0}\left(\mathbb{R}_{+}, B\right) \rightarrow C\left(\mathbb{R}_{+} \cup\{+\infty\}, B\right) \rightarrow B \rightarrow 0$.

We have a commuting diagram

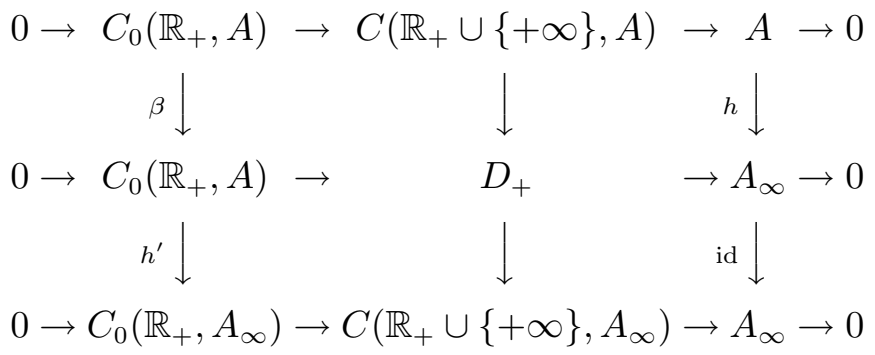

where $\beta: C_{0}\left(\mathbb{R}_{+}, A\right) \rightarrow C_{0}\left(\mathbb{R}_{+}, A\right)$ is given by $(\beta(f))(t)=\alpha_{t}(f(t))$ for every continuous function $f: \mathbb{R}_{+} \rightarrow A$ and $h^{\prime}: C_{0}\left(\mathbb{R}_{+}, A\right) \rightarrow C_{0}\left(\mathbb{R}_{+}, A_{\infty}\right)$ is given by $\left(h^{\prime}(f)\right)(t)=h_{t}(f(t))$ for every continuous function $f: \mathbb{R}_{+} \rightarrow$ $A$ (recall that $h_{t}=\alpha_{\infty}^{-t} \circ h$ ). As $\beta$ is homotopic to the identity among $C_{0}\left(\mathbb{R}_{+}\right)$-linear endomorphisms of $C_{0}\left(\mathbb{R}_{+}, A\right)$, the compositions $h^{*}(\varphi)$ defines the identity element of $E(A, A)$; as $h^{\prime}$ is homotopic to the map $f \mapsto h \circ f$ among $C_{0}\left(\mathbb{R}_{+}\right)$-linear homomorphisms of $C_{0}\left(\mathbb{R}_{+}, A\right)$ into $C_{0}\left(\mathbb{R}_{+}, A_{\infty}\right), h_{*}(\varphi)$ defines the identity element of $E\left(A_{\infty}, A_{\infty}\right)$.

It follows from the six term exact sequence of $E$-theory that $D$ is $E$ contractible. By Connes' analogue of the Thom isomorphism it follows that $D \rtimes \mathbb{R}$ is $E$-contractible and by Theorem $3.5, \mathcal{T}_{\alpha}$ is also $E$-contractible.

\section{References}

[1] A. Connes, An Analogue of the Thom Isomorphism for crossed product by an action of $\mathbb{R}$, Advances in Math., 39 (1981), 31-55.

[2] A. Connes and N. Higgson, Deformation, morphismes asymptotiques et $K$-Théorie bivariante, C.R. Acad. Sci. Paris, 311(I) (1990), 101-106.

[3] Duncan and Barnes, $C^{*}$-Algebras of Certain Inverse Semigroups.

[4] R. Exel, Circle Actions on $C^{*}$-algebras, Partial Automorphism and a Generalized Pimsner-Voiculescu Exact Sequence, to appear.

[5] T. Fack and G. Skandalis, Connes' Analogue of the Thom Isomorphism for the Kasparov Groups, Invert. Math., 64 (1981), 7-14.

[6] G.G. Kasparov, The operator $K$-functor and extensions of $C^{*}$-algebras, Math. U.S.S.R. Izv, 16 (1981), 513-572.

[7] - Hilbert $C^{*}$-modules, Theorem of Steinspring and Voiculescu, J. Operator Theory, 4 (1980), 133-150. 
[8] G. Peterson, $C^{*}$-Algebras and their Automorphism Groups, Academic Press, London, 1979.

[9] M. Pimsner, A class of $C^{*}$-algebras generalizing both Cuntz-Krieger algebras and crossed products by $\mathbb{Z}$, Fields Inst. Comm., 12, Amer. Math. Soc., Providence, RI, 1997.

[10] M. Pimsner and D. Voiculescu, Exact Sequences for K-groups and Ext-groups of certain crossed product $C^{*}$-Algebras, J. Operator Theory, 4 (1980), 93-118.

[11] G. Skandalis, Une notion de nucléarite en K-Théorie, K-Theory, (1988), 549-574.

Received March 29, 1996.

UNIVERSITY OF SASKATCHEWAN

SASKATOON SK S7N 5E6, CANADA

AND

EQUiPE D'Alain CONNES

College de France

75005 PARIS, FRANCE 